Swarthmore College

Works

\title{
Pretransitional Optical Activity In Chiral Smectic Liquid Crystals
}

K. C. Frame

J. L. Walker

Peter J. Collings

Swarthmore College, pcollin1@swarthmore.edu

Follow this and additional works at: https://works.swarthmore.edu/fac-physics

Part of the Physics Commons

Let us know how access to these works benefits you

\section{Recommended Citation}

K. C. Frame, J. L. Walker, and Peter J. Collings. (1991). "Pretransitional Optical Activity In Chiral Smectic Liquid Crystals". Molecular Crystals and Liquid Crystals. Volume 198, Issue 1. 91-97. DOI: 10.1080/ 00268949108033386

https://works.swarthmore.edu/fac-physics/221

This work is brought to you for free by Swarthmore College Libraries' Works. It has been accepted for inclusion in Physics \& Astronomy Faculty Works by an authorized administrator of Works. For more information, please contact myworks@swarthmore.edu. 
Mol. Cryst. Liq. Cryst., 1991, Vol. 198, pp. 91-97

Reprints available directly from the publisher

Photocopying permitted by license only

(c) 1991 Gordon and Brexch Science Publishers S.A.

Printed in the United States of America

\title{
Pretransitional Optical Activity in Chiral Smectic Liquid Crystals
}

\author{
K. C. FRAME; J. L. WALKER and P. J. COLLINGS† \\ Department of Physics, Kenyon College, Gambier, OH 43022
}

(Received July 25, 1990)

Pretransitional optical activity measurements in smectic liquid crystals of both low and high chirality are reported. In the low chirality sample, $p$-( $n$-(decyloxybenzylidene)-p-amino-(2-methylbutyl)cinnamate (DOBAMBC), the results indicate that the optical activity in both the isotropic phase just above the smectic $A$ phase and in the smectic $A$ phase just above the smectic $C^{*}$ phase can be explained in terms of a Landau-deGennes free energy with no coupling between the chiral modes. In the high chirality sample, 1-methylheptyl $4^{\prime}-\left[\left(4^{\prime \prime}\right.\right.$-tetradecyloxyphenyl)propioloyloxy]biphenyl-4-carboxylate (14P1M7), the data in the isotropic phase above the recently discovered smectic $\mathrm{A}^{*}$ phase cannot be explained in this way, even when coupling between the chiral modes is considered. Whether this is due to some feature of the smectic $A^{*}$ phase or is simply the result of strong coupling between the orientational and positional order parameters is a question which remains unanswered.

Keywords: liquid crysials, chiral phases, optical activity, phase transitions

\section{INTRODUCTION}

Recent theoretical ${ }^{1-4}$ and experimental ${ }^{5-8}$ work has demonstrated that the thermodynamic fluctuations which take place near transitions in chiral liquid crystals display a richness not present in non-chiral systems. Most of these investigations have concentrated on the fluctuations which take place in the isotropic phase, just above the transition to one of the blue phases. The results show (1) that these fluctuations can be quite strong with a complex temperature and wavelength dependence, and (2) that optical activity is a very sensitive probe into the nature of these fluctuations.

Very little work has been done in systems where the transition from the isotropic phase is to a smectic phase. In an effort to understand how these fluctuations are affected by the presence of short-range smectic order, we conducted optical activity experiments in the isotropic phase just above the transition to both the non-chiral smectic A phase ard the recently discovered chiral smectic $A$ phase. In an effort to understand the nature of the fluctuations when long-range smectic order is present, we also measured the optical activity in the smectic $A$ phase, just above

† Present address: Department of Physics \& Astronomy, Swarthmore College, Swarthmore, PA 19081. 
the transition to the chiral smectic $C$ phase. The results reported here agree with the findings of the single previous investigation in a system of low chirality, ${ }^{9}$ but raise new questions concerning our understanding of fluctuations in highly chiral smectic liquid crystals.

\section{THEORY}

As is customary in Landau-deGennes free energy calculations, the orientational order parameter is represented by the anisotropic part of the dielectric tensor, $\varepsilon_{\mathrm{t}}$. Since it is a symmetric tensor with zero trace, it can be represented in terms of five independent modes or basic tensors. The $m=0$ mode is nematic-like (nonchiral), the $m= \pm 1$ modes describe conical spiral modes, and the $m= \pm 2$ modes are planar spiral modes. The sense of the chirality (right- or left-handed) is indicated by the sign of $m$. The nematic phase $(\mathrm{N})$ is the simplest structure, and can be represented by the $m=0$ mode alone. The chiral nematic phase $\left(N^{*}\right)$ can be described by the appropriate combination of two modes, the $m=0$ and $m=-2$ modes for a right-handed helical structure and the $m=0$ and $m=+2$ modes for a left-handed chiral nematic. The wavevector, $q$, is zero for the $m=0$ mode and $4 \pi /$ pitch for the $m= \pm 2$ modes. The chiral smectic $C$ phase $\left(C^{*}\right)$ in general requires three modes for its description, but the amount of each depends on the tilt angle. Figure 1 shows the amplitude of each mode, $\left|\varepsilon_{m}\right|$, as a function of tilt angle for a left-handed $\mathrm{C}^{*}$ liquid crystal. In this case $q$ is zero for the $m=0$ mode and $2 \pi /$ pitch for the other modes. Since only the orientation order is being described, the nematic and chiral nematic phases can be seen as the limiting cases were the tilt angle equals $0^{\circ}$ and $90^{\circ}$, respectively.

The theory for fluctuations in the isotropic phase has been reviewed recently ${ }^{6}$

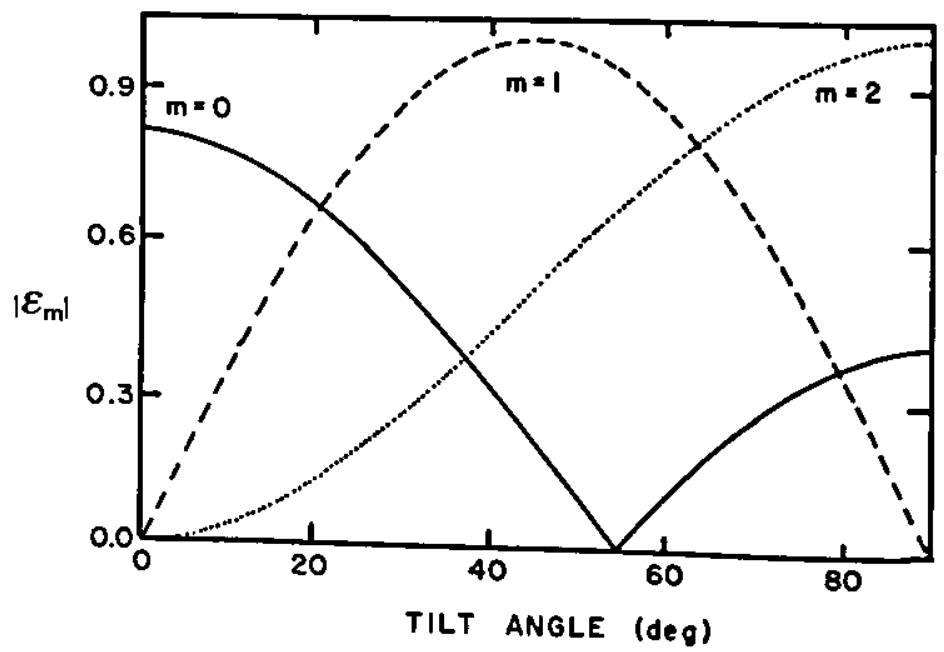

FIGURE 1 Relative amplitudes of the three modes in a left-handed chiral smectic $C$ phase ( $C^{*}$ ) as is the same as in Reference 3 . 
and will only be summarized here. The Landau-deGennes free energy is written in terms of the five modes, and if only terms up to second order are retained, each mode contributes to the free energy independently. Each mode possesses a tem-. perature at which the free energy for that mode vanishes, with the order $m=2$; $1,0,-1$, and -2 as the temperature decreases (or the reverse, depending on whether the liquid crystal is left-handed or right-handed). Only the $m=1$ and $m$ $=-1$ modes contribute to the optical activity in the isotropic phase, so applying the equipartition theorem and integrating over all wavevectors results in the prediction that the optical activity, $\theta$, should tend to diverge at the higher of the $m$ $=1$ or $m=-1$ second order transition temperatures with an exponent equal to $-1 / 2$,

$$
\theta=A /\left(T-T_{1}^{*}\right)^{1 / 2}+\theta_{0}
$$

where $T$ is the temperature and $A, T_{1}^{*}$, and $\theta_{0}$ are constants. The sign of the pretransitional optical activity is the same as the optical activity of the compound when dissolved in a solvent at low concentration (positive for dextrorotatory compounds and minus for levorotatory compounds). These predictions have been verified in liquid crystal systems of low chirality. ${ }^{10,11}$

If terms higher than second order are retained in the free energy express, coupling between the two modes with the highest second order transition temperatures (either $m=1$ and $m=2$ or $m=-1$ and $m=-2$, depending on the sense of chirality), produces an expression for the optical activity which reaches a maximum at a temperature slightly higher than the transition to the liquid crystal phase. This prediction has been verified qualitatively in highly chiral liquid crystals, but there seems to be some quantitative discrepancies between the theory and experiment. . $^{2.6 .7}$

If the transition to the liquid crystal phase is to a smectic phase, then fluctuations involving short-range positional order should occur along with the fluctuations involving orientational order. Any coupling between the positional and orientational order parameters should affect the fluctuations of the $m=1$ and $m=-1$ modes, which in turn should influence the pretransitional optical activity. Just as in the case of coupling between the chiral modes themselves, one would expect the coupling to be strongest in highly chiral systems.

Likewise, fluctuations of these modes in the smectic A phase as the smectic $\mathrm{C}^{*}$ phase is approached should be significantly affected by the presence of long-range positional order. This last case has been theoretically examined by Demikhov, Dolganov, and Filev. ${ }^{9}$ They use the Landau-deGennes free energy appropriate to the $A$ to $\mathrm{C}^{*}$ transition and consider the case where light propagates along the director in the smectic $A$ phase. In their calculations, they assume the correlation length for orientational fluctuations in the smectic A phase is much less than both the wavelength and the pitch of the $C^{*}$ phase. As in the case of fluctuations in the isotropic phase, the optical activity is predicted to diverge at a second order transition temperature with an exponent of $-1 / 2$. However, due to the different nature of the fluctuations in the smectic A phase, the optical activity should have the opposite sign as in the isotropic phase. 


\section{EXPERIMENT}

The low chirality smectic liquid crystal used in this study was (S)-(+)-p-(n-decyloxybenzylidene)-p-amino-(2-methylbutyl)cinnamate (DOBAMBC), with a pitch in the $C^{*}$ phase around $4000 \mathrm{~nm} .{ }^{12}$ This is the same compound used by Demikhov, Dolganov, and Filev in their initial investigation, ${ }^{9}$ and our desire was to repeat their experiments with a higher degree of precision. DOBAMBC was obtained from Frinton Laboratories; it was recrystallized once from ethanol for the measurements in the isotropic phase. The high chirality smectic liquid crystal studied was (R)-(+)-1-methylheptyl 4'-[(4"-tetradecyloxyphenyl) propioloxy]biphenyl-4carboxylate (14P1M7), with a $\mathrm{C}^{*}$ pitch of less than $500 \mathrm{~nm} .{ }^{13}$ This material possesses the recently discovered smectic $A^{*}$ phase between the isotropic and $C^{*}$ phases, ${ }^{13.14}$ and was kindly supplied to us by J. Goodby. It was used without further purification.

The optical rotation measurement system is of extremely high precision $\left( \pm 0.001^{\circ}\right)$. Light from either a HeNe or Argon Ion laser is linearly polarized and then passes through a Faraday Effect modulator. The modulated light then passes through the sample, then a second polarizer in a computer-controlled rotation stage, before finally striking the silicon diode detector, the output of the detector is fed into a lock-in amplifier, which also supplies the signal to the amplifier driving the Faraday Effect Modulator. With this experimental arrangement, the optical rotation angle is simply the angle between the two polarizers when the output of the lock-in amplifier crosses zero. The high precision is achieved by having the computer scan the second polarizer through this angle in steps of $0.01^{\circ}$. Linear interpolation then determines the zero crossing angle with a standard deviation of typically $0.001^{\circ}$.

The sample is contained between two pieces of glass in an Instec HS-1 hot stage. The temperature of the hot stage is maintained to within $0.001 \mathrm{~K}$, and the temperature inhomogeneity across the illuminated part of the sample is less than 0.01

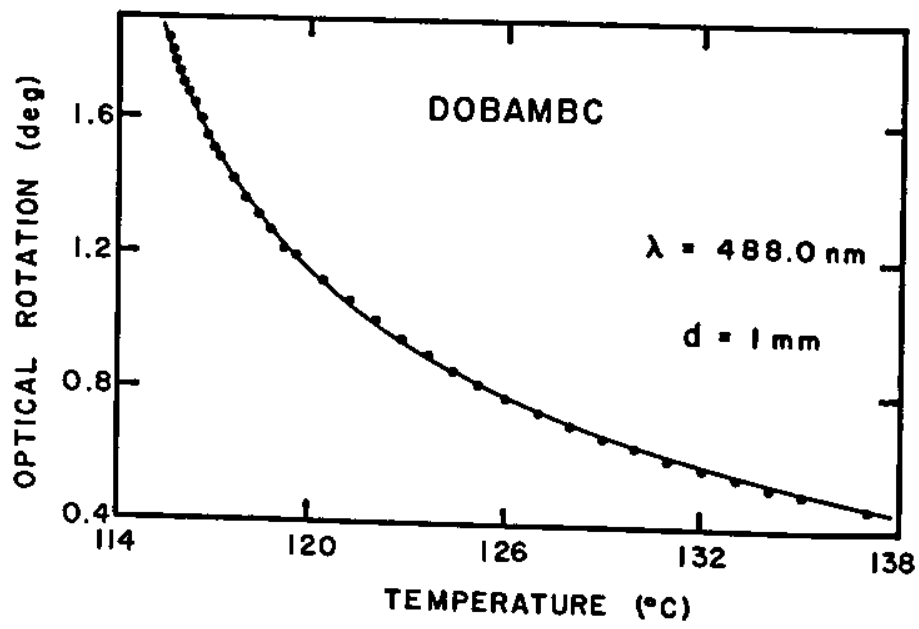

FIGURE 2 Optical rotation in the isotropic phase of DOBAMBC. The wavelength is $488.0 \mathrm{~nm}$ and the thickness of the sample is $1 \mathrm{~mm}$. The line is the least squares fit to Equation (1). 


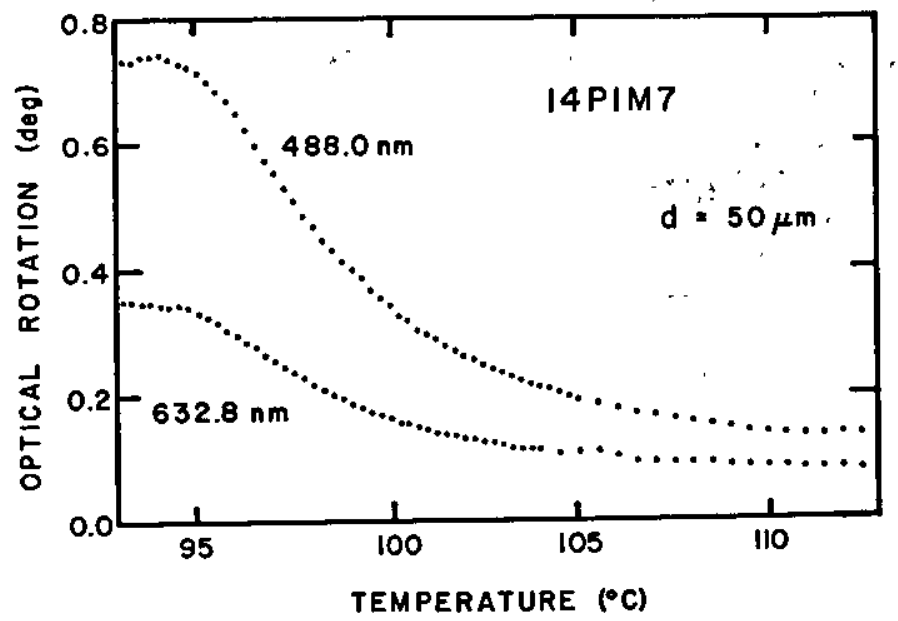

FIGURE 3 Optical rotation above the chiral smectic A phase ( $A^{*}$ ) of 14P1M7. The data for two wavelengths are given. The thickness of the sample is $50 \mu \mathrm{m}$.

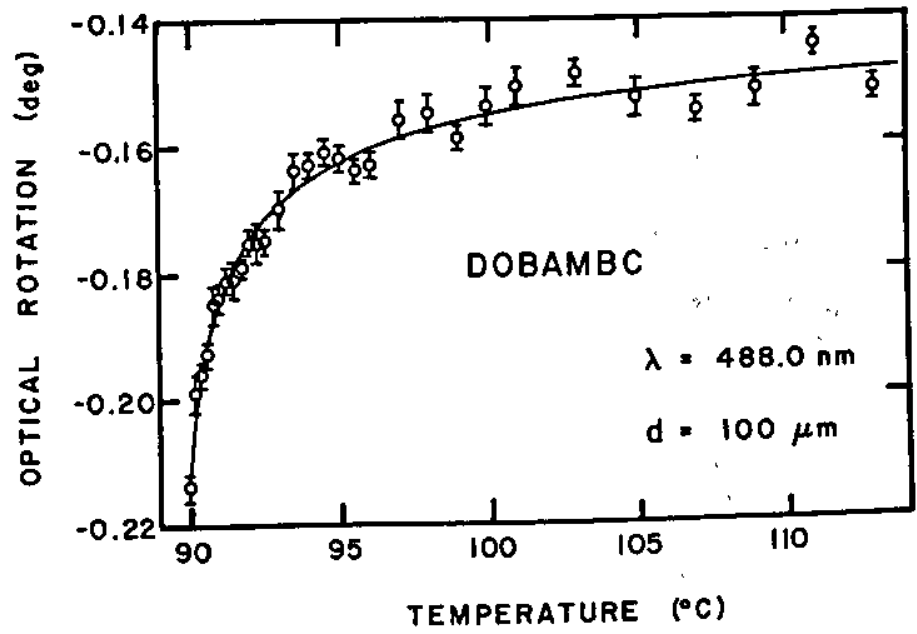

FIGURE 4 Optical rotation for light propagating parallel to the director in the smectic A phase (A) of DOBAMBC. The wavelength is $488.0 \mathrm{~nm}$ and the thickness of the sample is $100 \mu \mathrm{m}$. The line is the least squares fit to Equation (1).

$\mathrm{K}$. The thickness of the sample is maintained by a spacer placed between the two pieces of glass.

The optical activity in the isotropic phase of DOBAMBC is shown in Figure 2, where it is evident that the data closely follow the predicted temperature dependence. The temperature at which the data tends to diverge is $4.1 \pm 0.2 \mathrm{~K}$ below the transition to the smectic A phase. These results are very similar to those of Demikhov, Dolganov, and Filev, ${ }^{9}$ and indicate that the fluctuations of the chiral modes are not affected by whatever short-range smectic order is present. 
The results for a system with much higher chirality (14P1M7) are very different, as is evident from Figure 3. Notice that the optical activity does not possess the same temperature dependence as in DOBAMBC. The general shape of the data is reminiscent of what occurs in highly chiral liquid crystals when coupling between the modes is present. ${ }^{5-7}$ However, attempts to fit the data to the functional dependence typical of highly chiral liquid crystals fail miserably. The magnitude of the optical activity is also much larger than measurements in the isotropic phase of any other liquid crystals. The ratio of the optical activity at the two wavelengths used in the experiment is about 1.9 and does not vary from this value by more than $\pm 10 \%$ over the temperature range investigated. If measurements performed in the isotropic phase of a highly chiral but non-smectogenic compound are extrapolated to these two wavelengths (the data were taken between 700 and 1100 $\mathrm{nm}$ ), the ratio only varies by $\pm 5 \%$ from the value 1.9 .8 This seems to indicate that the optical activity in $14 \mathrm{P} 1 \mathrm{M} 7$ is due to chiral fluctuations, but the presence of short-range smectic order and high chirality appears to produce some very different effects.

Finally, the optical activity in the smectic A phase of DOBAMBC is shown in Figure 4. Homeotropic alignment was obtained by coating the glass with octadecyltriethoxysilane. As predicted theoretically, the sign of the optical activity is opposite from its sign in the isotropic phase. As evident from Figure 4 , the data follow the theoretical curve, with a divergent temperature $0.4 \pm 0.1 \mathrm{~K}$ below the transition to the $\mathrm{C}^{*}$ phase. In addition to verifying the measurements of Demikhov, Dolganov, and Filev, ${ }^{9}$ these results provide an even more stringent test of the theory.

\section{DISCUSSION}

The data presented here demonstrate that the pretransitional optical activity in smectic liquid crystals can be understood on the same basis as non-smectic liquid crystals if the chirality is not too high. This is true not only for fluctuations in the isotropic phase, but also for fluctuations in the smectic $A$ phase. However, there are experimental and theoretical reasons why the situation in highly chiral smectic liquid crystals may be quite different.

First of all, Filev argues that the chiral modes should show the effect of coupling in the smectic $A$ phase as well as the isotropic phase. ${ }^{4}$ The experimental results seem to indicate that DOBAMBC is not chiral enough to see such an effect. Similar measurements in highly chiral systems with an $\mathrm{A}-\mathrm{C}^{*}$ transition are planned in our laboratory and should give an indication of whether the theory is correct or not.

Second, the origin of the optical activity immediately above the $A^{*}$ phase is still an open question. Although the experimental results reported here give every indication that fluctuations above the transition cause the optical activity, one must proceed with caution. For example, DSC and heat capacity measurements on 14P1M7 show a small, broad peak just above the transition to the isotropic phase. ${ }^{13,14}$ In fact, the temperature at which the optical activity decreases fastest occurs at the same temperature as the center of the DSC and heat capacity peaks. If the optical 
activity is due to fluctuations, then these fluctuations are large enough to show up in thermal measurements. This is not true for other compounds, even the highly chiral ones. Could this optical activity be due to the presence of long-range orientational order in a narrow temperature interval above the $A^{*}$ phase? The data presented here seem to argue against such a possibility, since the pitch of such a phase would have to be greater than the wavelength of light in the sample in order for the optical activity to be positive. But a phase with a large pitch would not produce the ratio of optical activity at the two wavelengths typical of pretransitional measurements as is the case for 14P1M7. More theoretical and experimental work will be required to demonstrate whether there is something unique about the transition from the $A^{*}$ to isotropic phase, or if coupling between the smectic and orientational order parameters produces extremely strong fluctuations in highly chiral liquid crystals.

\section{Acknowledgment}

These results are based on work supported by a William and Flora Hewlitt Foundation Grant of the Research Corporation (Grant No. C-2430) and by Grant No. DMR-8906010 of the National Science Foundation. The assistance of J. W. Goodby in providing the 14P1M7, J. S. Patel in supplying the alignment agent, and J. C. Sosnowski in obtaining the data is deeply appreciated.

\section{References}

1. S. A. Brazovskii and S. G. Dmitriev, Zh. Eksp. Teor. Fiz., 69, 979 (1975); Sov. Phys. JETP, 42, 497 (1976).

2. V. K. Dolganov, S. P. Krylova and V. M. Filev, Zh. Eksp. Teor. Fiz., 78, 2343 (1980); Sov. Phys. JETP, 51, 1177 (1980).

3. D. Bensimon, E. Domany and S. Shrikman, Phys. Rev. A, 28, 427 (1983).

4. V. M. Filev, Pis'ma Zh. Eksp. Teor. Fiz., 37, 589 (1983); JETP Lett., 37, 703 (1983).

5. E. I. Demikhov and V. K. Dolganov, Pis'ma Zh. Eksp. Teor. Fiz., 38, 368 (1983); JETP Lett., 38, 368 (1983).

6. P. R. Battle, J. D. Miller and P. J. Collings, Phys. Rev. A, 36, 369 (1987).

7. F. Vanweert, W. Demol and W. Van Dael, Liq. Cryst., 5, 853 (1989).

8. J. E. Wyse, J. Ennis and P. J. Collings, Phys. Rev. Lett., 62, 1045 (1989).

9. E. 1. Demikhov, V. K. Dolganov and V. M. Filev, Pis'ma Zh. Eksp. Teor. Fiz., 37, 305 (1983); JETP Lett., 37, 361 (1983).

10. J. Cheng and R. B. Meyer, Phys. Rev. A, 9, 2744 (1974).

11. M. B. Atkinson and P. J. Collings, Mol. Cryst. Liq. Cryst., 136, 141 (1986).

12. L. A. Beresnev, L. M. Blinov, M. A. Osipov and S. A. Pikin, Mol. Cryst. Liq. Cryst., 158A, 112 (1988).

13. J. W. Goodby, M. A. Waugh, S. M. Stein, E. Chin, R. Pindak and J. S. Patel, Nature, 337, 449 (1989); J. Am. Chem. Soc., 111, 8119 (1989).

14. C. C. Huang, D. S. Lin, J. W. Goodby, M. A. Waugh, S. M. Stein and E. Chin, Phys. Rev. A, 40, 4153 (1989). 